\title{
Essential Oil Composition of Cirsium arvense L. Produced in Different Climate and Soil Properties
}

\author{
Narges Amiri ${ }^{\odot}$, Mehrab Yadegari ${ }^{\odot *}$ and Behzad Hamedi \\ Department of Agronomy and Medicinal Plants, Faculty of Agriculture, Islamic Azad University \\ Shahrekord Branch, Po.Box:166. Shahrekord, Iran
}

(Received June 28, 2017; Revised September 25, 2017; Accepted September 29, 2017)

\begin{abstract}
This study investigated the quantitative and qualitative effects of climate and soil properties on essential oil content and composition of Cirsium arvense L. (Asteraceae), which is an important medicinal plant. Root, stem, and leaf tissues were collected from plants found in four regions (Shahrekord, Farsan, Chelgerd and Ardal) of the Chaharmahal and Bakhtiari Province, Iran. GC/MS analysis revealed that the main constituents of essential oils from the various populations were nonadecane, $\beta$-citronellol, camphor, heneicosane and phytol. The highest levels of nonadecane (40.1-42\%) and camphor (18.1-18.4\%) were obtained from roots collected from the Chelgerd region, the most levels of $\beta$-citronellol (24.9-25.01\%) were obtained from leaves from the Chelgerd region, and the most levels of heneicosane (14.4-15.6\%) and phytol (11.8-12.58\%) were obtained from stems from the Shahrekord region. The most amount of essential oil (0.34-0.33\%) was obtained from roots growing in clay soil in the Chelgerd region. Both climate and soil properties had significant effects on the essential oil of $C$. arvense. The highestt essential oil contents were obtained from plants growing in clay soil, which seemed to have a greater capacity to hold water and nutrients, both of which promote plant growth and essential oil production.
\end{abstract}

Keywords: $\beta$-citronellol; camphor; Cirsium arvense; nonadecane. (C) 2018 ACG Publications. All rights reserved.

\section{Introduction}

Cirsium arvense L. (Asteraceae) is a perennial plant that grows in Iran and other countries. The genus Cirsium has been used as a flavouring agent, especially for dairy foods (yoghurt and cheese) and meat, by the indigenous people of the Chaharmahal and Bakhtiari province of southwestern Iran [1]. The plant is also used as food and for its medicinal properties. Extracts of Cirsium arvense produce a variety of pharmacological effects, including the inhibition of cholesterol biosynthesis [2-5]. Cirsium arvense is an erect, rhizomatous perennial thistle that is usually $0.5-1.0 \mathrm{~m}$ tall and changes its morphology in response to environmental conditions [6]. The species is invasive in prairies and other grasslands in the Midwest and Great Plains, as well as in riparian areas in the intermountain west, and is particularly troublesome in the northwest and north-central states of the USA [2,3]. The use of plants for treating ailments dates back several centuries. However, herbal medicine usually relies on tradition and may or may not be supported by empirical data. The belief that natural medicines are safer than synthetic drugs has recently increased and has induced tremendous growth in phyto-pharmaceutical usage [4]. C. arvense needs lower temperatures and a

\footnotetext{
*Corresponding author: mehrab_yadegari@yahoo.com Phone:098-913-3814318 Fax:098-383-3361093
} 
specific photoperiod to form generative stems and bloom, and though the plant is drought tolerant after establishment, moisture and soil nutrition are necessary for better yields [7]. Herb and spice products are widely used in folk medicine, food flavoring, and in the food industry [8]. In recent years, concern about the safety of synthetic compounds has increased interest in the use of natural substances and has encouraged more detailed studies on plant-derived substances. The quality and quantity of essential oils from medicinal plants are mainly influenced by genetic and environmental factors [9]. Environmental variations can influence the phytochemical properties of plants. For example, the artemisinin content of Artemisia annua [10], antioxidant content of vegetables [11], essential oil composition and antimicrobial properties of wild mint [12], and essential oil content of Origanum vulgare [13] increase with decreasing latitude. Altitude affects the morphology, epidermis, and anatomy of Pinus roxburghii needles [14], and significantly affects variation between durum wheat landraces [15]. Plants of the genus Cirsium are characterized by a high content of flavonoid compounds. Previously high content of linarin (5,7-dihydroxy-4-methoxyflavone, 7-0-[0- $\alpha$-Lrhamnopyranosyl- $\beta$-D-glucopyranoside) from leaves [16], apigenin, luteolin, 3-O-methyl kaempferol, cosmosiin, and acacetin 7- $\beta$-D-glucopyranosid uronic acid from flowers [17] have been detected. "Recently, some volatiles that attract both floral herbivores and pollinators were detected including 2phenylethanol, methyl salicylate, p-anisaldehyde, benzaldehyde, benzyl alcohol, phenylacetaldehyde, linalool, furanoid linalool oxides (E and Z), and dimethyl salicylate. Single compounds (and one isomer) set out in scent-baited water-bowl traps trapped over 10 species of pollinators and 16 species of floral herbivores. The two dominant components of the fragrance blend of $C$. arvense, benzaldehyde and phenylacetaldehyde, trapped both pollinators and florivores. Other compounds attracted either pollinators or florivores. Florivores of $C$. arvense appear to use floral scent compounds as kairomones, by advertising to pollinators" [18].

This study used gas chromatograph with flame ionization detector (GC/FID) and gas chromatography linked to a mass spectrometer show the main components (GC/MS) to analyse the effects of climate and soil on the phytochemical and morphological characteristics of $C$. arvense in Chaharmahal and Bakhtiari Province of Iran over two seasons.

\section{Materials and Methods}

\subsection{Sample Collection}

To determine the best environmental conditions and tissue for producing high-quality $C$. arvense, three types of tissue (root, stem, and leaf) were collected from plants found in four regions (Shahrekord, Farsan, Chelgerd, and Ardal) of Chaharmahal and Bakhtiari Province of southwest Iran in April-July 2016-2017, using a randomized complete design with three replicates per region. Plant material was collected from natural populations in each region. The climatic and soil characteristics of the sampling regions are listed in Tables 1 and 2. After sampling plants from each region, the root length, stem length, leaf area indices and total dry matter of the samples were measured.

Table 1. Climatic properties of regions of Chaharmahal and Bakhtiari Province, Iran

\begin{tabular}{lcccc}
\hline Characters & \multicolumn{3}{c}{ Region } \\
\cline { 2 - 5 } & Shahrekord & Farsan & Chelgerd & Ardal \\
\hline Average Annual precipitation $(\mathrm{mm})$ & 380 & 516.9 & 1480 & 500 \\
Average of annual temperature $\left(\mathrm{C}^{\circ}\right)$ & 11.7 & 12.1 & 9.5 & 18.4 \\
Average Maximum temperature $\left(\mathrm{C}^{\circ}\right)$ & 23.9 & 24.5 & 22.1 & 27.8 \\
Average Minimum temperature $\left(\mathrm{C}^{\circ}\right)$ & -1.8 & -1.4 & -5.1 & 4 \\
Height $(\mathrm{m})$ & 2060 & 2072 & 2390 & 1388 \\
Latitude and Longitude & $32^{0} 19^{\prime} \mathrm{N}-50^{0} 51^{\prime} \mathrm{E}$ & $32^{0} 26^{\prime} \mathrm{N}-50^{0} 56^{\prime} \mathrm{E}$ & $32^{0} 40^{\prime} \mathrm{N}-50^{0} 26^{\prime} \mathrm{E}$ & $31^{0} 99^{\prime} \mathrm{N}-50^{0} 66^{\prime} \mathrm{E}$ \\
\hline
\end{tabular}


Table 2. Physico-chemical properties of experimental soil $(0-30 \mathrm{~cm})$ in four regions of Chaharmahal and Bakhtiari Province, Iran

\begin{tabular}{|c|c|c|c|c|c|}
\hline \multirow[t]{2}{*}{ Year } & \multirow[t]{2}{*}{ Characters } & \multicolumn{4}{|c|}{ Region } \\
\hline & & Shahrekord & Farsan & Chelgerd & Ardal \\
\hline \multirow{12}{*}{2016} & $\mathrm{~N}_{\text {total }}(\%)$ & 0.153 & 0.119 & 0.186 & 0.046 \\
\hline & Organic Mat (\%) & 0.66 & 0.31 & 0.78 & 0.23 \\
\hline & $\mathrm{pH}$ & 8.03 & 7.49 & 7.66 & 7.71 \\
\hline & $\mathrm{P}(\mathrm{mg} / \mathrm{kg})$ & 15.3 & 27.1 & 35.7 & 17.6 \\
\hline & K (mg/kg) & 328 & 288 & 621 & 162 \\
\hline & $\mathrm{Ca}(\mathrm{mg} / \mathrm{kg})$ & 4.74 & 4.11 & 3.81 & 4.3 \\
\hline & $\operatorname{Mn}(\mathrm{mg} / \mathrm{kg})$ & 9.23 & 10.27 & 12.36 & 8.81 \\
\hline & $\mathrm{Fe}(\mathrm{mg} / \mathrm{kg})$ & 3.1 & 4.17 & 6.55 & 2.96 \\
\hline & $\mathrm{Cu}(\mathrm{mg} / \mathrm{kg})$ & 0.63 & 0.77 & 0.86 & 0.55 \\
\hline & Zn (mg/kg) & 0.72 & 0.86 & 0.96 & 0.79 \\
\hline & EC (ds/m) & 0.77 & 1.7 & 0.45 & 0.45 \\
\hline & Texture & Clay Loam & Clay Loam & Clay & Clay \\
\hline \multirow[t]{12}{*}{2017} & $\mathrm{~N}_{\text {total }}(\%)$ & 0.14 & 0.12 & 0.191 & 0.036 \\
\hline & Organic Mat (\%) & 0.59 & 0.34 & 0.91 & 0.21 \\
\hline & $\mathrm{pH}$ & 7.9 & 7.2 & 7.52 & 7.61 \\
\hline & $\mathrm{P}(\mathrm{mg} / \mathrm{kg})$ & 14.7 & 25.3 & 32.6 & 16.9 \\
\hline & K (mg/kg) & 311 & 273 & 614 & 172 \\
\hline & $\mathrm{Ca}(\mathrm{mg} / \mathrm{kg})$ & 4.9 & 4.2 & 3.7 & 4.5 \\
\hline & Mn (mg/kg) & 8.14 & 9.3 & 10.1 & 7.9 \\
\hline & $\mathrm{Fe}(\mathrm{mg} / \mathrm{kg})$ & 2.8 & 3.91 & 6.12 & 2.81 \\
\hline & $\mathrm{Cu}(\mathrm{mg} / \mathrm{kg})$ & 0.61 & 0.62 & 0.77 & 0.49 \\
\hline & Zn (mg/kg) & 0.69 & 0.82 & 0.91 & 0.71 \\
\hline & $\mathrm{EC}(\mathrm{ds}(\mathrm{m})$ & 0.65 & 0.93 & 0.42 & 0.41 \\
\hline & Texture & Clay Loam & Clay Loam & Clay & Clay \\
\hline
\end{tabular}

\subsection{Treatments}

Fresh aerial $C$. arvense tissues were dried for 10 days at room temperature $\left(25 \pm 5^{\circ} \mathrm{C}\right)$, and then ground to fine a powder using a Moulinex food processor. The essential oil was extracted by heating $50 \mathrm{~g}$ of each tissue (root, stem or leaf) in a $2 \mathrm{~L}$ flask with $1 \mathrm{~L}$ water using a heating jacket at $100^{\circ} \mathrm{C}$ for $3 \mathrm{~h}$ in a Clevenger-type apparatus, according to the procedures outlined by the British Pharmacopoeia. Voucher specimens (20078-TUH) were those described by Mozaffarian [19] and were deposited in Herbarium of the Center of Agricultural and Natural Resources of Chaharmahal and Bakhtiari Province, Shahrekord, Iran.

\subsection{Essential Oil Extraction}

The essential oil content was determined by distilling tissues in Clevenger type apparatus. One thousand gram of plant tissue were placed in $6 \mathrm{~L}$ Clevenger-type distillation apparatuses and distilled for $5 \mathrm{~h}$ in $3 \mathrm{~L}$ of pure water. The quantities of plant oils obtained at the end of distillation were measured in $\mathrm{mL}$ and ratios $(\% \mathrm{w} / \mathrm{w})$ were determined by multiplying oil content with oil density (i.e., $\left.0.858 \mathrm{~g} . \mathrm{cm}^{3}\right)$. All the essential oil samples were dried over hydrous sodium sulphate, and stored at $4^{\circ}$ $\mathrm{C}$ until $\mathrm{GC}$ and $\mathrm{GC}-\mathrm{MS}$ analysis.

\subsection{Essential Oil Analysis}

Ground GC analysis was done using an Agilent Technologies 7890 GC equipped with FID and a HP-5MS 5\% capillary column. The carrier gas was helium at a flow rate of $0.8 \mathrm{~mL} / \mathrm{min}$. Initial column temperature was $60^{\circ} \mathrm{C}$ and programmed to increase at $4{ }^{\circ} \mathrm{C} / \mathrm{min}$ to $280{ }^{\circ} \mathrm{C}$. The split ratio was 
40:1. The injector temperature was set at $300{ }^{\circ} \mathrm{C}$. The purity of helium gas was $99.99 \%$ and $0.1 \mathrm{~mL}$ samples were injected manually in the split mode. GC-MS analyses were carried out on a Thermo Finnigan Trace $2000 \mathrm{GC} / \mathrm{MS}$ system equipped with a HP-5MS capillary column $(30 \mathrm{~m} \times 0.25 \mathrm{~mm}$ i.d., film thickness $0.25 \mu \mathrm{m}$ ). Oven temperature was held at $120^{\circ} \mathrm{C}$ for $5 \mathrm{~min}$ and then programmed to reach $280{ }^{\circ} \mathrm{C}$ at a rate of $10^{\circ} \mathrm{C} / \mathrm{min}$. Detector temperature was $260^{\circ} \mathrm{C}$ and injector temperature was $260{ }^{\circ} \mathrm{C}$. The composition of the essential oil were identified by comparing retention indices relative to a series of $n$-alkanes $\left(\mathrm{C}_{7}-\mathrm{C}_{24}\right)$; retention times and mass spectra were those of authentic samples from Wiley library [20].

\section{Results and Discussion}

The morphology, essential oil content, and essential oil composition of the populations differed significantly ( $p \leq 0.01$ ) (Tables 3 and 4). Twenty-five compounds, accounted for $84.2-91.9 \%$ and $82.89-99.9 \%$ of the total oil content, (Table 5). The five most abundant components, which composed $65.2-81.1 \%$ and $64.1-79.5 \%$ of the essential oil content in first and second years, respectively, were camphor, $\beta$-citronellol, heneicosane, nonadecane and phytol. Furthermore, based on dry weight, the yield $(\mathrm{w} / \mathrm{w})$ of the obtained essential oils ranged from $0.24-0.34 \%$ to $0.28-0.33 \%$ in the first and second years, respectively (Table 5). Meanwhile, the least abundant components were 3buten-2-one, 2-pentadecanone, bicyclo[5.2.0]nonane, 1,6-octadien-3-ol, 6-octen-1-ol, 2naphthalenemethanol and cyclohexanone (0-6.6\% to 0-7.8\%). The highest levels of nonadecane (40.1$42 \%$ ) and camphor (18.1-18.4\%) were obtained from the roots of plants found in the Chelgerd region, the highest levels of $\beta$-citronellol (24.9-25.01\%) were obtained from the leaves of plants found in the Chelgerd region, and the highest levels of heneicosane (14.4-15.6\%) and phytol (11.8-12.58\%) were obtained from stems of plants found in the Shahrekord region. The greatest essential oil content $(0.34-$ $0.33 \%$ ) was obtained from plant roots plants growing in clay soil in the Chelgerd region (Table 5 ). The greatest levels of camphor, $\beta$-citronellol, and nonadecane were observed for plants growing in clay soil in the Chelgerd region at elevations $>2300 \mathrm{~m}$. At higher elevations, the most abundant essential oil components were camphor, $\beta$-citronellol, heneicosane, nonadecane, cis-2,6-dimethyl-2,6-octadiene and phytol. Both climate and plant tissue affect the yield of essential oils from medicinal plants. In this study, the percentages of organic matter, phosphorus, potassium, manganese, iron, copper and zinc increased with increasing altitude. At higher elevation, greater precipitation also increased plant growth (Tables 1 and 2). There were significant differences among the essential oil compositions of the various samples, and there was a positive correlation between the essential oil content and components contained in each of the three analysed plant tissues. Contents of the main essential oil components (camphor, $\beta$-citronellol, heneicosane, nonadecane, and phytol) were significantly correlated with the essential oil content of each plant tissue. Correlation coefficients between the essential oil content and composition of each plant tissue suggested that the essential oil content of each plant tissue usually had a negative effect on the essential oil content of the other tissues, and that the main essential oil components of each plant tissue negatively affected the main essential oil components of the other plant tissues (Table 6).

In this study, nonadecane was the most abundant essential oil component of $C$. arvense, even though this was not reported previously. Other compounds such as $\beta$-citronellol, camphor, heneicosane and phytol. $\beta$-citronellol is used in perfumes and insect repellents and as a mite attractant [21]. Camphor is believed to be toxic to insects and is sometimes used as a repellent [22]. Benzaldehyde and phenylacetaldehyde in $C$. arvense have previously been reported previously, to trap pollinators and florivores. Other compounds such as 2-phenylethanol, methyl salicylate, panisaldehyde, benzaldehyde, benzyl alcohol, phenylacetaldehyde, linalool, furanoid linalool oxides (E and Z), and dimethyl salicylate attracted either pollinators or florivores [18]. The oils of Cirsium creticum (Lam.) (4-ethyl guaiacol, hexadecanoic acid, (E)-b-damascenone, dihydroactinidiolide, 4vinyl guaiacol) and Carduus nutans L. (hexadecanoic acid, hexahydrofarnesylacetone, heptacosane, 4vinyl guaiacol, pentacosane and eugenol) have weak inhibitory activity against seed germination and radical elongation of Raphanus sativus L. (radish), Lactuca sativa L. (lettuce), and Lepidium sativum L. (garden cress) [23]. 
Table 3. ANOVA (mean of squares) results of regional effects on morphological characteristics of Cirsium arvense

\begin{tabular}{|c|c|c|c|c|c|c|c|c|c|}
\hline \multirow[t]{2}{*}{ S.O.V } & \multirow[t]{2}{*}{ D.F } & \multicolumn{4}{|c|}{ First Year } & \multicolumn{4}{|c|}{ Second Year } \\
\hline & & T.D.M & S.L & R.L & L.A.I & T.D.M & S.L & R.L & L.A.I \\
\hline Treatment & 3 & $151.55^{* *}$ & $61.13^{* *}$ & $3.79^{\mathrm{ns}}$ & $13.91^{* *}$ & $148.2^{* *}$ & $44.17^{* *}$ & $11.82^{* *}$ & $24.8^{* * 1}$ \\
\hline Error & 8 & $\begin{array}{l}45.18 \\
12.42\end{array}$ & $\begin{array}{c}3.47 \\
11.25\end{array}$ & $\begin{array}{l}6.56 \\
8.63\end{array}$ & $\begin{array}{l}2.41 \\
3.25\end{array}$ & $\begin{array}{c}35.2 \\
4.8\end{array}$ & $\begin{array}{l}9.9 \\
7.7\end{array}$ & $\begin{array}{l}4.2 \\
5.5\end{array}$ & $\begin{array}{c}4.5 \\
6.63\end{array}$ \\
\hline
\end{tabular}

†S.O.V: Source Of Variation, D.F: Degree of Freedom, T.D.M: Total Dry Matter, S.L: Stem Length, R.L: Root Length, L.A.I: Leaf Area Indices ns: Not Significant, *and** : Significant at $\mathrm{P} \leq 0.05$ and $\mathrm{P} \leq 0.01$ levels respectively.

Table 4. ANOVA (mean of squares) of regional effects on the main essential oil component in Cirsium arvense roots, stem, and leaves.

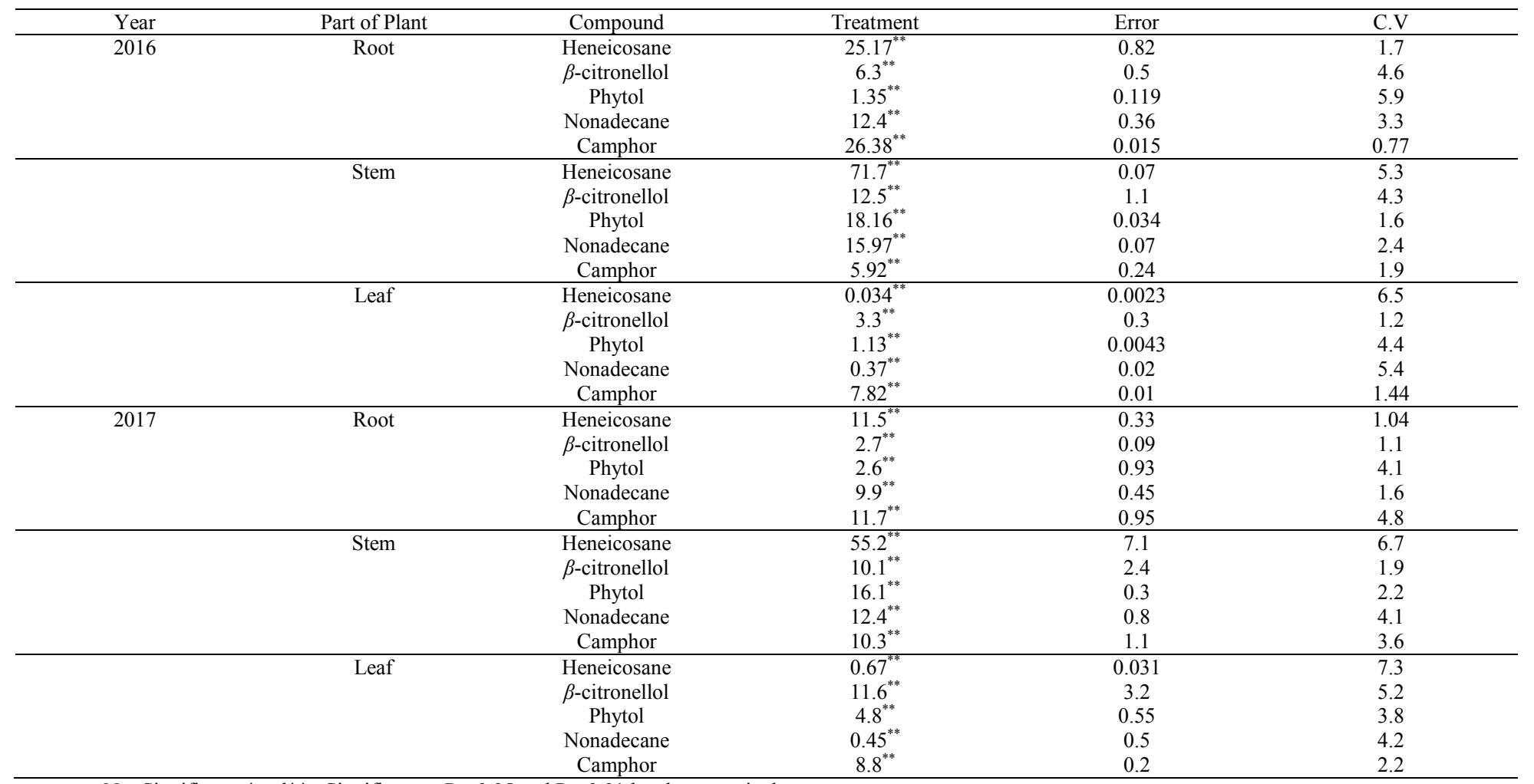

ns: Not Significant, *and** : Significant at $\mathrm{P} \leq 0.05$ and $\mathrm{P} \leq 0.01$ levels respectively. 
Table 5. Chemical composition (\%) of the essential oils of Cirsium arvense in two years (in each row, numbers have same word, are same group).

\begin{tabular}{|c|c|c|c|c|c|c|c|c|c|c|c|c|c|c|}
\hline \multirow[t]{2}{*}{ 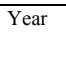 } & \multirow[t]{2}{*}{ Compound } & \multirow[t]{2}{*}{ RI } & \multirow{2}{*}{$\begin{array}{c}\text { Shahrekord } \\
\times \text { Root }\end{array}$} & \multirow{2}{*}{$\begin{array}{c}\text { Shahrekord } \\
\times \text { Stem }\end{array}$} & \multirow{2}{*}{$\begin{array}{c}\text { Shahrekord } \\
\times \text { Leaf }\end{array}$} & \multirow{2}{*}{$\begin{array}{l}\text { Farsan } \\
\times \text { Root }\end{array}$} & \multirow{2}{*}{$\begin{array}{l}\text { Farsan } \\
\times \text { Stem }\end{array}$} & \multirow{2}{*}{$\begin{array}{l}\text { Farsan } \\
\times \text { Leaf }\end{array}$} & \multirow{2}{*}{$\begin{array}{c}\text { Chelgerd } \\
\times \text { Root }\end{array}$} & \multirow[t]{2}{*}{ Chelgerd $\times$ Stem } & \multirow[t]{2}{*}{ Chelgerd $\times$ Leaf } & \multirow[t]{2}{*}{ Ardal $\times$ Root } & \multirow{2}{*}{$\begin{array}{l}\text { Ardal } \\
\times \text { Stem }\end{array}$} & \multirow{2}{*}{$\begin{array}{l}\text { Ardal } \\
\times \text { Leaf }\end{array}$} \\
\hline & & & & & & & & & & & & & & \\
\hline \multirow[t]{27}{*}{2016} & Camphor & 1145 & $15.1 \pm 0.5 \mathrm{~b}$ & $7.4 \pm 0.3 \mathrm{e}$ & $5.5 \pm 0.4 \mathrm{f}$ & $15.2 \pm 0.2 \mathrm{~b}$ & $9.1 \pm 0.1 \mathrm{~d}$ & $7.7 \pm 0.1 \mathrm{e}$ & $18.1 \pm 0.5 \mathrm{a}$ & $9.1 \pm 0.2 \mathrm{~d}$ & $7.7 \pm 0.2 \mathrm{e}$ & $11.5 \pm 0.5 \mathrm{c}$ & $7.6 \pm 0.1 \mathrm{e}$ & $5.1 \pm 0.1 \mathrm{f}$ \\
\hline & Cyclohexanone, 5-methyl & 1191 & $0.0001 \pm 0.00001$ & $0.001 \pm 0.0001$ & $0.0001 \pm 0.00001$ & $1.1 \pm 0.001$ & $1.2 \pm 0.01$ & $0.65 \pm 0.01$ & $3.2 \pm 0.002$ & $1.1 \pm 0.003$ & $1.2 \pm 0.01$ & $0.0001 \pm 0.00002$ & $0.0001 \pm 0.00002$ & $0.0001 \pm 0.00002$ \\
\hline & $\beta$-Citronellol & 1210 & $1.4 \pm 0.4 \mathrm{f}$ & $12.2 \pm 0.5 \mathrm{~d}$ & $15.2 \pm 0.5 \mathrm{c}$ & $9.1 \pm 0.2 \mathrm{e}$ & $18.1 \pm 0.2 \mathrm{~b}$ & $24.8 \pm 0.2 \mathrm{a}$ & $9.1 \pm 0.1 \mathrm{e}$ & $18.2 \pm 0.5 \mathrm{~b}$ & $24.9 \pm 0.8 \mathrm{a}$ & $2.3 \pm 0.5 \mathrm{f}$ & $14.4 \pm 0.5 \mathrm{c}$ & $15.1 \pm 0.2 \mathrm{c}$ \\
\hline & 1,6-Octadien-3-ol & 1234 & $0.12 \pm 0.02$ & $0.00003 \pm 0.00006$ & $0.00001 \pm 0.000001$ & 4.2. \pm 0.2 & $2.1 \pm 0.02$ & $1.1 \pm 0.1$ & $1.1 \pm 0.3$ & $1.8 \pm 0.03$ & $2.5 \pm 0.01$ & $0.13 \pm 0.001$ & $0.0001 \pm 0.00002$ & $0.0001 \pm 0.00001$ \\
\hline & 6-Octen-1-ol, & 1248 & $0.11 \pm 0.01$ & $0.0006 \pm 0.00001$ & $0.00001 \pm 0.000001$ & $0.11 \pm 0.01$ & $0.001 \pm 0.0001$ & $0.0002 \pm 0.00001$ & $0.0001 \pm 0.00001$ & $0.0001 \pm 0.00001$ & $0.0002 \pm 0.00001$ & $0.11 \pm 0.01$ & $0.00001 \pm 0.000001$ & $0.00001 \pm 0.00001$ \\
\hline & 2,6-Octadien-1-ol & 1355 & $0.2 \pm 0.01$ & $0.1 \pm 0.01$ & $0.04 \pm 0.001$ & $0.11 \pm 0.01$ & $0.002 \pm 0.0001$ & $0.001 \pm 0.0001$ & $0.1 \pm 0.001$ & $0.001 \pm 0.0001$ & $0.002 \pm 0.0004$ & $0.11 \pm 0.003$ & $0.11 \pm 0.001$ & $0.08 \pm 0.006$ \\
\hline & Bicyclo[5.2.0]nonane & 1394 & $0.02 \pm 0.001$ & $0.00005 \pm 0.000002$ & $0.00001 \pm 0.000005$ & $0.02 \pm 0.003$ & $0.001 \pm 0.0002$ & $0.0002 \pm 0.00002$ & $0.001 \pm 0.0005$ & $0.003 \pm 0.0001$ & $0.0004 \pm 0.00001$ & $0.03 \pm 0.001$ & $0.00002 \pm 0.000002$ & $0.00002 \pm 0.00008$ \\
\hline & 2-Naphthalenemethanol & 1468 & $0.02 \pm 0.005$ & $0.00008 \pm 0.000006$ & $0.00006 \pm 0.000003$ & $0.1 \pm 0.002$ & $0.001 \pm 0.0001$ & $0.0001 \pm 0.00005$ & $0.02 \pm 0.003$ & $0.003 \pm 0.0005$ & $0.0002 \pm 0.00004$ & $0.014 \pm 0.001$ & $0.00001 \pm 0.000005$ & $0.00001 \pm 0.000007$ \\
\hline & Pentadecane & 1500 & $0.01 \pm 0.001$ & $0.001 \pm 0.0004$ & $0.0003 \pm 0.00003$ & $0.77 \pm 0.07$ & $0.88 \pm 0.1$ & $0.55 \pm 0.005$ & $1.8 \pm 0.003$ & $1.01 \pm 0.1$ & $1.1 \pm 0.02$ & $0.01 \pm 0.003$ & $0.006 \pm 0.0003$ & $0.0002 \pm 0.00003$ \\
\hline & $\alpha$-Terpinolen & 1510 & $0.9 \pm 0.01$ & $0.8 \pm 0.03$ & $0.8 \pm 0.01$ & $0.9 \pm 0.02$ & $0.82 \pm 0.011$ & $0.01 \pm 0.001$ & $0.83 \pm 0.01$ & $0.6 \pm 0.01$ & $0.009 \pm 0.0001$ & $1.01 \pm 0.008$ & $0.05 \pm 0.001$ & $0.81 \pm 0.001$ \\
\hline & Cyclohexanone & 1597 & $0.001 \pm 0.0001$ & $0.00005 \pm 0.000001$ & $0.00002 \pm 0.000003$ & $0.9 \pm 0.001$ & $2.2 \pm 0.01$ & $2.61 \pm 0.01$ & $1.1 \pm 0.01$ & $3.3 \pm 0.011$ & $2.2 \pm 0.03$ & $0.001 \pm 0.0001$ & $0.00002 \pm 0.000001$ & $0.00001 \pm 0.000005$ \\
\hline & $\begin{array}{l}\text { Cis-2,6-Dimethyl- } \\
\text { 2,6-octadiene }\end{array}$ & 1611 & $2.2 \pm 0.1$ & $1.4 \pm 0.5$ & $14.4 \pm 0.5$ & $1.2 \pm 0.1$ & $8.4 \pm 0.5$ & $14.8 \pm 0.6$ & $1.01 \pm 0.02$ & $9.01 \pm 0.1$ & $11.1 \pm 0.1$ & $3.9 \pm 0.2$ & $1.7 \pm 0.1$ & $3.5 \pm 0.05$ \\
\hline & Trans-Caryophyllene & 1632 & $1.1 \pm 0.01$ & $1.3 \pm 0.04$ & $0.94 \pm 0.04$ & $0.11 \pm 0.02$ & $0.88 \pm 0.02$ & $0.45 \pm 0.03$ & $0.11 \pm 0.04$ & $0.89 \pm 0.01$ & $0.4 \pm 0.002$ & $1.4 \pm 0.02$ & $0.8 \pm 0.02$ & $0.8 \pm 0.1$ \\
\hline & Trans-Geraniol & 1645 & $1.45 \pm 0.1$ & $1.2 \pm 0.02$ & $0.96 \pm 0.01$ & $0.86 \pm 0.02$ & $4.4 \pm 0.02$ & $1.1 \pm 0.1$ & $0.14 \pm 0.002$ & $5.5 \pm 0.1$ & $1.4 \pm 0.1$ & $1.5 \pm 0.1$ & $0.98 \pm 0.02$ & $1.2 \pm 0.2$ \\
\hline & $\beta$-Lonone & 1672 & $1.4 \pm 0.1$ & $0.94 \pm 0.1$ & $0.87 \pm 0.1$ & $0.1 \pm 0.01$ & $0.001 \pm 0.001$ & $0.00002 \pm 0.000001$ & $0.12 \pm 0.02$ & $0.002 \pm 0.0001$ & $0.01 \pm 0.001$ & $0.87 \pm 0.02$ & $0.78 \pm 0.1$ & $0.91 \pm 0.02$ \\
\hline & Heptadecane & 1701 & $2.3 \pm 0.1$ & $0.85 \pm 0.01$ & $1.2 \pm 0.1$ & $0.02 \pm 0.002$ & $0.04 \pm 0.001$ & $0.00002 \pm 0.000001$ & $0.02 \pm 0.001$ & $0.05 \pm 0.001$ & $0.03 \pm 0.004$ & $2.7 \pm 0.1$ & $0.94 \pm 0.1$ & $1.1 \pm 0.1$ \\
\hline & 9-Nonadecene & 1811 & $1.4 \pm 0.1$ & $0.9 \pm 0.01$ & $0.89 \pm 0.01$ & $0.03 \pm 0.005$ & $0.002 \pm 0.0001$ & $0.00008 \pm 0.000001$ & $0.03 \pm 0.001$ & $0.0001 \pm 0.00002$ & $0.0002 \pm 0.00002$ & $1.1 \pm 0.1$ & $0.9 \pm 0.01$ & $0.9 \pm 0.01$ \\
\hline & $n$-Nonadecane & 1901 & $2.1 \pm 0.02$ & $1.4 \pm 0.01$ & $1.1 \pm 0.1$ & $2.2 \pm 0.4$ & $2.1 \pm 0.1$ & $2.1 \pm 0.03$ & $2.2 \pm 0.1$ & $2.2 \pm 0.01$ & $2.4 \pm 0.01$ & $1.9 \pm 0.01$ & $1.6 \pm 0.04$ & $1.4 \pm 0.04$ \\
\hline & Heneicosane & 2109 & $2.1 \pm 0.1 \mathrm{~d}$ & $14.4 \pm 0.02 \mathrm{a}$ & $0.99 \pm 0.05 \mathrm{e}$ & $6.82 \pm 0.6 \mathrm{~b}$ & $1.92 \pm 0.1 \mathrm{~d}$ & $1.1 \pm 0.1 \mathrm{e}$ & $7.7 \pm 0.3 \mathrm{~b}$ & $2.2 \pm 0.2 \mathrm{~d}$ & $1.1 \pm 0.1 \mathrm{e}$ & $4.9 \pm 0.2 \mathrm{c}$ & $5.5 \pm 0.4 \mathrm{c}$ & $0.9 \pm 0.2 \mathrm{e}$ \\
\hline & Nonadecane & 2112 & $38.2 \pm 2.3 \mathrm{a}$ & $33.2 \pm 3.3 \mathrm{~b}$ & $25.1 \pm 1.4 \mathrm{~d}$ & $38.1 \pm 3.2 \mathrm{a}$ & $30.2 \pm 1.2 \mathrm{c}$ & $18.4 \pm 1.1 \mathrm{f}$ & $40.1 \pm 1.1 \mathrm{a}$ & $30.1 \pm 1.1 \mathrm{c}$ & $17.4 \pm 1.2 \mathrm{f}$ & $37.7 \pm 1.5 \mathrm{~b}$ & $35.5 \pm 2.4 \mathrm{~b}$ & $30.2 \pm 1.4 \mathrm{c}$ \\
\hline & 3-Buten-2-one & 2111 & $0.01 \pm 0.001$ & $0.9 \pm 0.1$ & $0.89 \pm 0.1$ & $0.11 \pm 0.001$ & $0.00001 \pm 0.000001$ & $0.00002 \pm 0.000002$ & $0.1 \pm 0.002$ & $0.00001 \pm 0.000001$ & $0.00001 \pm 0.000002$ & $0.02 \pm 0.001$ & $0.85 \pm 0.001$ & $0.8 \pm 0.002$ \\
\hline & 2-Pentadecanone & 2115 & $0.02 \pm 0.001$ & $0.002 \pm 0.0002$ & $0.0004 \pm 0.00006$ & $0.01 \pm 0.001$ & $0.00001 \pm 0.000001$ & $0.00003 \pm 0.000001$ & $0.01 \pm 0.001$ & $0.00001 \pm 0.000001$ & $0.00001 \pm 0.000001$ & $0.02 \pm 0.001$ & $0.002 \pm 0.0002$ & $0.0003 \pm 0.00001$ \\
\hline & $\begin{array}{l}\text { 1,2-Benzenedicarboxylic } \\
\text { acid }\end{array}$ & 2117 & $3.3 \pm 0.2$ & $2.2 \pm 0.1$ & $3.8 \pm 0.04$ & $0.02 \pm 0.004$ & $0.0002 \pm 0.00001$ & $0.00002 \pm 0.000003$ & $0.03 \pm 0.001$ & $0.0002 \pm 0.00001$ & $0.0001 \pm 0.00002$ & $3.9 \pm 0.1$ & $5.5 \pm 0.1$ & $4.4 \pm 0.04$ \\
\hline & Z-5-Nonadecene & 2118 & $4.4 \pm 0.1$ & $3.5 \pm 0.05$ & $4.1 \pm 0.1$ & $0.87 \pm 0.1$ & $0.92 \pm 0.02$ & $0.77 \pm 0.1$ & $1.1 \pm 0.1$ & $0.0005 \pm 0.00001$ & $1.3 \pm 0.03$ & $4.9 \pm 0.1$ & $3.8 \pm 0.1$ & $4.4 \pm 0.03$ \\
\hline & Phytol & 2120 & $9.9 \pm 0.1 \mathrm{~b}$ & $4.9 \pm 0.1 \mathrm{c}$ & $11.8 \pm 0.7 \mathrm{a}$ & $1.4 \pm 0.3 \mathrm{~d}$ & $1.1 \pm 0.1 \mathrm{~d}$ & $9.9 \pm 0.4 \mathrm{~b}$ & $0.66 \pm 0.3 \mathrm{~d}$ & $0.88 \pm 0.2 \mathrm{~d}$ & $9.92 \pm 0.3 \mathrm{~b}$ & $11.9 \pm 0.3 \mathrm{a}$ & $4.4 \pm 0.1 \mathrm{c}$ & $11.4 \pm 0.2 \mathrm{a}$ \\
\hline & Total & & 87.76 & 87.59 & 88.58 & 84.36 & 84.36 & 86.05 & 88.6 & 85.95 & 84.6 & 91.9 & 85.42 & 84.2 \\
\hline & $\begin{array}{l}\text { Essential oil content } \\
\text { (\%) }\end{array}$ & & $0.28 \pm 0.02 \mathrm{~b}$ & $0.29 \pm 0.01 \mathrm{~b}$ & $0.25 \pm 0.01 \mathrm{c}$ & $0.3 \pm 0.01 \mathrm{~b}$ & $0.28 \pm 0.02 \mathrm{~b}$ & $0.27 \pm 0.01 \mathrm{c}$ & $0.34 \pm 0.01 \mathrm{a}$ & $0.29 \pm 0.01 \mathrm{~b}$ & $0.31 \pm 0.02 \mathrm{a}$ & $0.29 \pm 0.01 \mathrm{~b}$ & $0.28 \pm 0.02 \mathrm{~b}$ & $0.24 \pm 0.01 \mathrm{c}$ \\
\hline \multirow[t]{11}{*}{2017} & Camphor & 1146 & $14.2 \pm 0.9 \mathrm{~b}$ & $6.9 \pm 0.8 \mathrm{f}$ & $5.9 \pm .03 \mathrm{f}$ & $18.3 \pm 0.3 \mathrm{a}$ & $9.2 \pm 0.1 \mathrm{~d}$ & $8.2 \pm 0.2 \mathrm{e}$ & $18.4 \pm 0.6 \mathrm{a}$ & $9.3 \pm 0.3 \mathrm{~d}$ & $8.4 \pm 0.4 \mathrm{e}$ & $12.5 \pm 0.6 \mathrm{c}$ & $6.9 \pm 0.1 \mathrm{f}$ & $5.2 \pm 0.1 \mathrm{~g}$ \\
\hline & Cyclohexanone, 5-methyl & 1190 & $0.00012 \pm 0.00001$ & $0.001 \pm 0.0001$ & $0.0005 \pm 0.00001$ & $4.1 \pm 0.001$ & $1.9 \pm 0.01$ & $0.95 \pm 0.001$ & $4.2 \pm 0.002$ & $2 \pm 0.003$ & $1.02 \pm 0.001$ & $0.00001 \pm 0.000002$ & $0.00001 \pm 0.000004$ & $0.00001 \pm 0.000005$ \\
\hline & $\beta$-Citronellol & 1210 & $1.8 \pm 0.3 \mathrm{~g}$ & $14 \pm 0.8 \mathrm{e}$ & $16.7 \pm 0.5 \mathrm{~d}$ & $9.5 \pm 0.3 \mathrm{f}$ & $19.2 \pm 0.3 \mathrm{c}$ & $24.01 \pm 0.4 \mathrm{a}$ & $9.6 \pm 0.2 \mathrm{f}$ & $20.1 \pm 0.7 \mathrm{~b}$ & $25.01 \pm 0.9 \mathrm{a}$ & $1.23 \pm 0.3 \mathrm{~g}$ & $13 \pm 0.9 \mathrm{e}$ & $16.2 \pm 0.9 \mathrm{~d}$ \\
\hline & 1,6-Octadien-3-ol & 1234 & $0.14 \pm 0.01$ & $0.00004 \pm 0.00005$ & $0.00002 \pm 0.000001$ & $5.2 \pm 0.1$ & $2.3 \pm 0.01$ & $2.1 \pm 0.1$ & $5.3 \pm 0.03$ & $2.9 \pm 0.04$ & $2.6 \pm 0.02$ & $0.13 \pm 0.001$ & $0.00001 \pm 0.000002$ & $0.00001 \pm 0.000001$ \\
\hline & 6-Octen-1-ol, & 1247 & $0.17 \pm 0.001$ & $0.00007 \pm 0.000005$ & $0.00002 \pm 0.0000001$ & $0.33 \pm 0.01$ & $0.002 \pm 0.0001$ & $0.0001 \pm 0.00001$ & $0.0003 \pm 0.00001$ & $0.0003 \pm 0.00002$ & $0.0004 \pm 0.00001$ & $0.15 \pm 0.01$ & $0.00001 \pm 0.000001$ & $0.00001 \pm 0.000002$ \\
\hline & 2,6-Octadien-1-ol & 1356 & $0.26 \pm 0.01$ & $0.17 \pm 0.01$ & $0.1 \pm 0.01$ & $0.21 \pm 0.03$ & $0.004 \pm 0.0001$ & $0.001 \pm 0.0002$ & $0.22 \pm 0.001$ & $0.005 \pm 0.0001$ & $0.0022 \pm 0.0004$ & $0.24 \pm 0.003$ & $0.16 \pm 0.05$ & $0.09 \pm 0.006$ \\
\hline & Bicyclo[5.2.0]nonane & 1393 & $0.03 \pm 0.002$ & $0.00008 \pm 0.000005$ & $0.00002 \pm 0.000007$ & $0.01 \pm 0.004$ & $0.001 \pm 0.0006$ & $0.0003 \pm 0.00007$ & $0.002 \pm 0.0005$ & $0.0032 \pm 0.0005$ & $0.00045 \pm 0.00006$ & $0.022 \pm 0.001$ & $0.00001 \pm 0.000007$ & $0.00001 \pm 0.000008$ \\
\hline & 2-Naphthalenemethanol & 1468 & $0.03 \pm 0.006$ & $0.00009 \pm 0.000007$ & $0.00009 \pm 0.000008$ & $0.11 \pm 0.004$ & $0.002 \pm 0.0001$ & $0.0003 \pm 0.00005$ & $0.12 \pm 0.003$ & $0.0034 \pm 0.0006$ & $0.0004 \pm 0.00008$ & $0.024 \pm 0.004$ & $0.00001 \pm 0.000007$ & $0.00001 \pm 0.000009$ \\
\hline & Pentadecane & 1500 & $0.02 \pm 0.008$ & $0.007 \pm 0.0008$ & $0.0004 \pm 0.00005$ & $1.1 \pm 0.09$ & $1.01 \pm 0.1$ & $0.81 \pm 0.009$ & $1.2 \pm 0.008$ & $1.02 \pm 0.1$ & $1.23 \pm 0.08$ & $0.012 \pm 0.007$ & $0.0065 \pm 0.0005$ & $0.00025 \pm 0.00004$ \\
\hline & $\alpha$-Terpinolen & 1510 & $1.02 \pm 0.02$ & $1.1 \pm 0.06$ & $1.2 \pm 0.08$ & $1.02 \pm 0.09$ & $0.99 \pm 0.01$ & $0.012 \pm 0.001$ & $1.03 \pm 0.05$ & $1.01 \pm 0.07$ & $0.014 \pm 0.005$ & $1.01 \pm 0.008$ & $0.99 \pm 0.006$ & $1.1 \pm 0.004$ \\
\hline & Cyclohexanone & 1595 & $0.002 \pm 0.0001$ & $0.00007 \pm 0.000002$ & $0.00004 \pm 0.000008$ & $1.2 \pm 0.004$ & $4.8 \pm 0.01$ & $4.1 \pm 0.01$ & $1.3 \pm 0.04$ & $4.99 \pm 0.05$ & $4.8 \pm 0.09$ & $0.001 \pm 0.0005$ & $0.00003 \pm 0.000001$ & $0.00002 \pm 0.000007$ \\
\hline
\end{tabular}


Table 5. Continued..

\begin{tabular}{|c|c|c|c|c|c|c|c|c|c|c|c|c|c|}
\hline Cis-2,6-Dimethyl- & 1610 & $4.3 \pm 0.1$ & $2.2 \pm 0.5$ & $14.9 \pm 0.8$ & $1.3 \pm 0.2$ & $9.2 \pm 0.3$ & $15.2 \pm 0.2$ & $1.4 \pm 0.04$ & $9.99 \pm 0.3$ & $15.9 \pm 0.2$ & $4.2 \pm 0.3$ & $2.1 \pm 0.1$ & $4.3 \pm 0.09$ \\
\hline \multicolumn{14}{|l|}{ 2,6-octadiene } \\
\hline Trans-Caryophyllene & 1632 & $2.84 \pm 0.08$ & $1 \pm 0.09$ & $1.01 \pm 0.07$ & $0.12 \pm 0.04$ & $0.98 \pm 0.01$ & $0.21 \pm 0.05$ & $0.13 \pm 0.07$ & $0.99 \pm 0.03$ & $0.3 \pm 0.001$ & $2.33 \pm 0.06$ & $0.95 \pm 0.06$ & $0.99 \pm 0.03$ \\
\hline Trans-Geraniol & 1645 & $2.25 \pm 0.1$ & $1.7 \pm 0.09$ & $2 \pm 0.08$ & $0.14 \pm 0.04$ & $6.2 \pm 0.03$ & $2.1 \pm 0.1$ & $0.16 \pm 0.001$ & $6.1 \pm 0.2$ & $2.99 \pm 0.2$ & $2.11 \pm 0.1$ & $1.68 \pm 0.09$ & $1.97 \pm 0.2$ \\
\hline$\beta$-Lonone & 1672 & $1.35 \pm 0.2$ & $1 \pm 0.1$ & $1.01 \pm 0.2$ & $0.12 \pm 0.04$ & $0.001 \pm 0.003$ & $0.00002 \pm 0.000005$ & $0.13 \pm 0.07$ & $0.001 \pm 0.005$ & $0.012 \pm 0.005$ & $1.1 \pm 0.04$ & $1 \pm 0.2$ & $0.99 \pm 0.03$ \\
\hline Heptadecane & 1700 & $2.6 \pm 0.1$ & $1 \pm 0.1$ & $1.36 \pm 0.1$ & $0.023 \pm 0.005$ & $0.048 \pm 0.005$ & $0.00003 \pm 0.000008$ & $0.025 \pm 0.005$ & $0.051 \pm 0.007$ & $0.032 \pm 0.007$ & $3.2 \pm 0.2$ & $1 \pm 0.1$ & $1.25 \pm 0.1$ \\
\hline 9-Nonadecene & 1811 & $1.65 \pm 0.2$ & $1 \pm 0.1$ & $1 \pm 0.2$ & $0.02 \pm 0.004$ & $0.001 \pm 0.001$ & $0.00009 \pm 0.000007$ & $0.04 \pm 0.002$ & $0.00012 \pm 0.00005$ & $0.0001 \pm 0.00005$ & $1.23 \pm 0.2$ & $0.92 \pm 0.1$ & $1 \pm 0.1$ \\
\hline$n$-Nonadecane & 1900 & $2.3 \pm 0.09$ & $2 \pm 0.09$ & $2 \pm 0.2$ & $3.01 \pm 0.2$ & $3.02 \pm 0.2$ & $2.22 \pm 0.09$ & $3.02 \pm 0.2$ & $3.03 \pm 0.1$ & $2.8 \pm 0.1$ & $2.23 \pm 0.1$ & $1.99 \pm 0.09$ & $1.99 \pm 0.08$ \\
\hline Heneicosane & 2109 & $2.9 \pm 0.3 \mathrm{~d}$ & $15.6 \pm 0.2 \mathrm{a}$ & $1 \pm 0.09 \mathrm{e}$ & $7.2 \pm 0.4 \mathrm{~b}$ & $2.2 \pm 0.4 \mathrm{~d}$ & $0.98 \pm 0.1 \mathrm{e}$ & $8.1 \pm 0.5 b$ & $2.4 \pm 0.5 \mathrm{~d}$ & $1.2 \pm 0.1 \mathrm{e}$ & $5.53 \pm 0.2 \mathrm{c}$ & $5.2 \pm 0.1 \mathrm{c}$ & $0.97 \pm 0.2 \mathrm{e}$ \\
\hline Nonadecane & 2110 & $41 \pm 0.9 \mathrm{a}$ & $34.2 \pm 3.3 \mathrm{c}$ & $28.1 \pm 1.8 \mathrm{~d}$ & $40 \pm 3.7 \mathrm{a}$ & $31.1 \pm 1.4 \mathrm{c}$ & $17.1 \pm 2.3 \mathrm{e}$ & $42 \pm 1.2 \mathrm{a}$ & $32.1 \pm 1.5 \mathrm{c}$ & $18.2 \pm 1.3 \mathrm{e}$ & $38.2 \pm 0.9 \mathrm{~b}$ & $33.2 \pm 2.6 \mathrm{c}$ & $27.2 \pm 2.8 \mathrm{~d}$ \\
\hline 3-Buten-2-one & 2111 & $0.02 \pm 0.001$ & $1 \pm 0.2$ & $1 \pm 0.1$ & $0.123 \pm 0.003$ & $0.00001 \pm 0.000002$ & $0.00002 \pm 0.000007$ & $0.16 \pm 0.004$ & $0.00001 \pm 0.000006$ & $0.00001 \pm 0.000009$ & $0.022 \pm 0.001$ & $0.99 \pm 0.001$ & $0.98 \pm 0.003$ \\
\hline 2-Pentadecanone & 2115 & $0.03 \pm 0.001$ & $0.004 \pm 0.0005$ & $0.0006 \pm 0.00008$ & $0.014 \pm 0.0009$ & $0.00001 \pm 0.000007$ & $0.00006 \pm 0.000003$ & $0.02 \pm 0.001$ & $0.00001 \pm 0.000002$ & $0.00001 \pm 0.000002$ & $0.025 \pm 0.001$ & $0.0026 \pm 0.0005$ & $0.00034 \pm 0.00002$ \\
\hline $\begin{array}{l}\text { 1,2-Benzenedicarboxylic } \\
\text { acid }\end{array}$ & 2117 & $4.9 \pm 0.2$ & $4.3 \pm 0.1$ & $3.98 \pm 0.09$ & $0.024 \pm 0.005$ & $0.0003 \pm 0.00001$ & $0.00004 \pm 0.000008$ & $0.033 \pm 0.001$ & $0.0004 \pm 0.00002$ & $0.0002 \pm 0.00001$ & $4.3 \pm 0.2$ & $4.2 \pm 0.1$ & $3.8 \pm 0.07$ \\
\hline Z-5-Nonadecene & 2118 & $5.7 \pm 0.1$ & $3.8 \pm 0.06$ & $4.12 \pm 0.1$ & $1.2 \pm 0.1$ & $1 \pm 0.06$ & $1 \pm 0.1$ & $1.6 \pm 0.1$ & $0.0006 \pm 0.00004$ & $1.9 \pm 0.09$ & $5.2 \pm 0.1$ & $3.4 \pm 0.1$ & $3.84 \pm 0.09$ \\
\hline Phytol & 2120 & $10.2 \pm 0.2 \mathrm{~b}$ & $5.36 \pm 0.1 \mathrm{c}$ & $12.58 \pm 0.7 \mathrm{a}$ & $1.2 \pm 0.1 \mathrm{~d}$ & $1.02 \pm 0.1 \mathrm{~d}$ & $11.2 \pm 0.9 \mathrm{a}$ & $1.4 \pm 0.6 \mathrm{~d}$ & $1.03 \pm 0.5 \mathrm{~d}$ & $11.89 \pm 0.8 \mathrm{a}$ & $12 \pm 0.4 \mathrm{a}$ & $5.2 \pm 0.1 \mathrm{c}$ & $12.36 \pm 0.3 \mathrm{a}$ \\
\hline Total & & 99.9 & 96.5 & 98.1 & 95.5 & 94.18 & 90.19 & 99.5 & 97.02 & 98.3 & 97.03 & 82.89 & 84.32 \\
\hline $\begin{array}{l}\text { Essential oil content } \\
\text { (\%) }\end{array}$ & & $0.29 \pm 0.03 \mathrm{ab}$ & $0.3 \pm 0.02 \mathrm{ab}$ & $0.295 \pm 0.01 \mathrm{ab}$ & $0.28 \pm 0.01 \mathrm{~b}$ & $0.3 \pm 0.05 \mathrm{ab}$ & $0.29 \pm 0.02 \mathrm{~b}$ & $0.33 \pm 0.02 \mathrm{a}$ & $0.285 \pm 0.02 \mathrm{~b}$ & $0.3 \pm 0.02 \mathrm{ab}$ & $0.29 \pm 0.01 \mathrm{~b}$ & $0.29 \pm 0.01 \mathrm{~b}$ & $0.25 \pm 0.02 \mathrm{c}$ \\
\hline
\end{tabular}


Table 6. Results of simple correlation between morphological properties, essential oil content and composition in Cirsium arvense in different regions and soil properties in the first year.

\begin{tabular}{|c|c|c|c|c|c|c|c|c|c|c|c|c|c|c|c|c|c|c|c|c|c|}
\hline \multirow[t]{22}{*}{$\begin{array}{c}\text { Total } \\
\text { dry } \\
\text { matter } \\
(22) \\
\end{array}$} & $\begin{array}{l}\text { Stem } \\
\text { length } \\
\text { (21) }\end{array}$ & $\begin{array}{c}\text { Root } \\
\text { lenght } \\
\text { (20) }\end{array}$ & $\begin{array}{l}\text { L.A.I } \\
\text { (19) }\end{array}$ & $\begin{array}{c}\text { Heneicosane } \\
\text { in leaves } \\
\text { (18) }\end{array}$ & $\begin{array}{c}\beta- \\
\text { citronellol } \\
\text { in leaves } \\
(17)\end{array}$ & $\begin{array}{c}\text { Phytol } \\
\text { in } \\
\text { leaves } \\
\text { (16) }\end{array}$ & $\begin{array}{c}\text { Nonadecane } \\
\text { in leaves } \\
(15)\end{array}$ & $\begin{array}{c}\text { Camphor } \\
\text { in leaves } \\
(14)\end{array}$ & $\begin{array}{l}\text { Heneicosane } \\
\text { in stem (13) }\end{array}$ & $\begin{array}{c}\beta- \\
\text { citronellol } \\
\text { in stem } \\
\text { (12) }\end{array}$ & $\begin{array}{c}\text { Phytol } \\
\text { in } \\
\text { stem } \\
\text { (11) }\end{array}$ & $\begin{array}{l}\text { Nonadecane } \\
\text { in stem (10) }\end{array}$ & $\begin{array}{l}\text { Camphor } \\
\text { in stem } \\
\text { (9) }\end{array}$ & $\begin{array}{c}\text { Heneicosane } \\
\text { of root (8) }\end{array}$ & $\begin{array}{c}\beta- \\
\text { citronellol } \\
\text { in root (7) }\end{array}$ & $\begin{array}{l}\text { Phytol } \\
\text { in } \\
\text { root } \\
(6)\end{array}$ & $\begin{array}{c}\text { Nonadecane } \\
\text { in root (5) }\end{array}$ & $\begin{array}{l}\text { Camphor } \\
\text { in root } \\
\text { (4) }\end{array}$ & $\begin{array}{c}\text { Essential } \\
\text { oil of } \\
\text { Leaves } \\
(3)\end{array}$ & $\begin{array}{c}\text { Essential } \\
\text { oil of } \\
\text { Stem (2) }\end{array}$ & $\begin{array}{c}\text { Essential } \\
\text { oil of } \\
\text { Root (1) }\end{array}$ \\
\hline & & & & & & & & & & & & & & & & & & & & - & -0.1 \\
\hline & & & & & & & & & & & & & & & & & & & - & 0.1 & -0.3 \\
\hline & & & & & & & & & & & & & & & & & & - & 0.2 & -0.1 & $0.5^{*}$ \\
\hline & & & & & & & & & & & & & & & & & - & 0.11 & $-0.5^{*}$ & -0.1 & $0.9^{* * *}$ \\
\hline & & & & & & & & & & & & & & & & - & -0.2 & 0.3 & -0.4 & 0.2 & 0.2 \\
\hline & & & & & & & & & & & & & & & - & $0.9^{* *}$ & $0.95^{* *}$ & 0.2 & $-0.5^{*}$ & 0.2 & $0.8^{* *}$ \\
\hline & & & & & & & & & & & & & & - & $0.9^{* * *}$ & $0.9^{* *}$ & $0.95^{* \prime}$ & 0.15 & 0.42 & 0.2 & -0.2 \\
\hline & & & & & & & & & & & & & - & $0.9^{* *}$ & $0.9^{* * *}$ & 0.4 & 0.4 & 0.17 & 0.41 & 0.3 & -0.2 \\
\hline & & & & & & & & & & & & - & $0.9^{* * *}$ & $0.9^{* *}$ & $0.9^{* * *}$ & 0.3 & 0.3 & -0.2 & $-0.6^{*}$ & $0.6^{*}$ & -0.2 \\
\hline & & & & & & & & & & & - & $0.9^{* * *}$ & $0.9^{* * *}$ & $-0.9^{* * *}$ & $-0.9^{* *}$ & 0.4 & 0.4 & 0.2 & 0.37 & $0.6^{*}$ & 0.3 \\
\hline & & & & & & & & & & - & $0.9^{* * *}$ & $0.9^{* * *}$ & $0.9^{* * *}$ & 0.3 & $-0.9^{* *}$ & $-0.9^{* * *}$ & 0.4 & -0.2 & $-0.7^{*}$ & $0.7^{*}$ & -0.2 \\
\hline & & & & & & & & & - & $0.9^{* * *}$ & $0.9^{* * *}$ & $0.9^{* * *}$ & $0.9^{* *}$ & $-0.9^{* * *}$ & $-0.9^{* * *}$ & 0.3 & 0.4 & 0.2 & -0.3 & $0.6^{*}$ & 0.2 \\
\hline & & & & & & & & - & $-0.9^{* * *}$ & $-0.9^{* * *}$ & $-0.9^{* * *}$ & $-0.9^{* *}$ & $-0.9^{* *}$ & $-0.9^{* * *}$ & 0.3 & $-0.9^{* * *}$ & $-0.6^{*}$ & $-0.7^{* *}$ & 0.37 & 0.3 & $-0.7^{* *}$ \\
\hline & & & & & & & - & 0.2 & -0.3 & -0.2 & $-0.9^{* *}$ & -0.3 & -0.3 & -0.2 & -0.3 & 0.2 & -0.2 & -0.1 & $0.7^{* * *}$ & -0.1 & 0.2 \\
\hline & & & & & & - & $0.6^{*}$ & $0.8^{* *}$ & $-0.9^{* * *}$ & $-0.8^{* *}$ & $-0.8^{* *}$ & $-0.8^{* *}$ & $-0.8^{* *}$ & $-0.8^{* * *}$ & $-0.8^{* *}$ & $-0.8^{* * *}$ & 0.4 & $0.6^{*}$ & -0.2 & $-0.6^{*}$ & 0.2 \\
\hline & & & & & - & $0.8^{* *}$ & 0.2 & $0.9^{* *}$ & $-0.9^{* *}$ & $-0.9^{* * *}$ & $-0.9^{* * *}$ & $-0.9^{* * *}$ & $-0.9^{* *}$ & $-0.6^{*}$ & 0.3 & $-0.9^{* * *}$ & $-0.6^{*}$ & $-0.8^{* *}$ & $0.7^{* *}$ & 0.2 & $-0.6^{*}$ \\
\hline & & & & - & 0.5 & 0.1 & 0.3 & 0.5 & 0.4 & -0.5 & -0.4 & 0.5 & $-0.8^{* *}$ & $-0.6^{*}$ & 0.5 & -0.5 & -0.2 & $0.65^{*}$ & 0.37 & $-0.6^{*}$ & -0.3 \\
\hline & & & - & $0.8^{* *}$ & $0.8^{* * *}$ & 0.3 & 0.4 & $0.8^{* *}$ & $0.6^{*}$ & $0.8^{* *}$ & $0.6^{*}$ & $0.7^{* * *}$ & $0.7^{* * *}$ & $0.8^{* *}$ & $0.7^{* * *}$ & $0.8^{* *}$ & $0.77^{* \prime}$ & $0.7^{* * *}$ & 0.36 & 0.3 & $0.7^{* * *}$ \\
\hline & & - & 0.1 & 0.1 & 0.1 & 0.1 & 0.1 & 0.1 & 0.1 & 0.1 & 0.1 & 0.1 & 0.1 & 0.1 & 0.1 & 0.1 & 0.1 & 0.1 & 0.1 & 0.2 & $0.6^{*}$ \\
\hline & - & 0.4 & $0.7^{* *}$ & 0.1 & 0.5 & 0.4 & 0.2 & 0.5 & 0.5 & 0.5 & 0.1 & 0.1 & 0.5 & 0.5 & 0.5 & $0.7^{* * *}$ & 0.4 & $0.6^{*}$ & 0.1 & 0.1 & 0.3 \\
\hline & 0.4 & 0.1 & 0.2 & 0.3 & $0.6^{*}$ & 0.35 & $0.7^{* *}$ & $0.6^{*}$ & 0.2 & $0.7^{* *}$ & 0.2 & $0.6^{*}$ & 0.2 & $0.6^{*}$ & $0.6^{*}$ & 0.3 & $0.8^{* *}$ & ${ }^{* *} 0.6$ & $0.8^{* *}$ & $0.7^{* * *}$ & $0.6^{* *}$ \\
\hline
\end{tabular}


Table 6.continued.

\begin{tabular}{|c|c|c|c|c|c|c|c|c|c|c|c|c|c|c|c|c|c|c|c|c|c|}
\hline \multirow[t]{21}{*}{$\begin{array}{c}\text { Total } \\
\text { dry } \\
\text { matter } \\
(22)\end{array}$} & $\begin{array}{l}\text { Stem } \\
\text { length } \\
(21)\end{array}$ & $\begin{array}{c}\text { Root } \\
\text { lenght } \\
\text { (20) }\end{array}$ & $\begin{array}{l}\text { L.A.I } \\
\text { (19) }\end{array}$ & $\begin{array}{c}\text { Heneicosane } \\
\text { in leaves } \\
(18)\end{array}$ & $\begin{array}{c}\beta- \\
\text { citronellol } \\
\text { in leaves } \\
(17)\end{array}$ & $\begin{array}{c}\text { Phytol } \\
\text { in } \\
\text { leaves } \\
(16)\end{array}$ & $\begin{array}{c}\text { Nonadecane } \\
\text { in leaves } \\
(15)\end{array}$ & $\begin{array}{c}\text { Camphor } \\
\text { in leaves } \\
(14)\end{array}$ & $\begin{array}{l}\text { Heneicosane } \\
\text { in stem (13) }\end{array}$ & $\begin{array}{c}\beta- \\
\text { citronellol } \\
\text { in stem } \\
\text { (12) }\end{array}$ & $\begin{array}{c}\text { Phytol } \\
\text { in } \\
\text { stem } \\
\text { (11) }\end{array}$ & $\begin{array}{l}\text { Nonadecane } \\
\text { in stem (10) }\end{array}$ & $\begin{array}{l}\text { Camphor } \\
\text { in stem } \\
\text { (9) }\end{array}$ & $\begin{array}{c}\text { Heneicosane } \\
\text { of root }(8)\end{array}$ & $\begin{array}{c}\beta- \\
\text { citronellol } \\
\text { in root (7) }\end{array}$ & $\begin{array}{l}\text { Phytol } \\
\text { in } \\
\text { root } \\
(6)\end{array}$ & $\begin{array}{c}\text { Nonadecane } \\
\text { in root (5) }\end{array}$ & $\begin{array}{l}\text { Camphor } \\
\text { in root } \\
\text { (4) }\end{array}$ & $\begin{array}{c}\text { Essential } \\
\text { oil of } \\
\text { Leaves } \\
\text { (3) }\end{array}$ & $\begin{array}{c}\text { Essential } \\
\text { oil of } \\
\text { Stem (2) }\end{array}$ & $\begin{array}{c}\text { Essential } \\
\text { oil of } \\
\text { Root (1) }\end{array}$ \\
\hline & & & & & & & & & & & & & & & & & & & & - & -0.2 \\
\hline & & & & & & & & & & & & & & & & & & & - & 0.2 & -0.2 \\
\hline & & & & & & & & & & & & & & & & & & - & 0.3 & -0.2 & $0.65^{*}$ \\
\hline & & & & & & & & & & & & & & & & & - & 0.2 & $-0.4^{*}$ & -0.1 & $0.8^{* *}$ \\
\hline & & & & & & & & & & & & & & & & - & -0.1 & 0.2 & -0.3 & 0.3 & 0.1 \\
\hline & & & & & & & & & & & & & & & - & $0.8^{* *}$ & $0.9^{* *}$ & $0.5^{*}$ & $-0.6^{* *}$ & 0.2 & $0.7^{* * *}$ \\
\hline & & & & & & & & & & & & & & - & $0.8^{* * *}$ & $0.7^{* * *}$ & $0.8^{* *}$ & 0.1 & 0.3 & 0.2 & -0.1 \\
\hline & & & & & & & & & & & & & - & $0.8^{* *}$ & $0.8^{* * *}$ & $0.5^{*}$ & $0.5^{*}$ & 0.1 & 0.3 & 0.3 & -0.1 \\
\hline & & & & & & & & & & & & - & $0.8^{* *}$ & $0.8^{* *}$ & $0.8^{* *}$ & 0.2 & 0.4 & -0.1 & $-0.7^{* *}$ & $0.5^{*}$ & -0.1 \\
\hline & & & & & & & & & & & - & $0.8^{* *}$ & $0.8^{* *}$ & $-0.7^{* * *}$ & $-0.7^{* *}$ & 0.2 & 0.3 & 0.1 & 0.3 & $0.4^{*}$ & 0.21 \\
\hline & & & & & & & & & & - & $0.8^{* *}$ & $0.7^{* *}$ & $0.7^{* *}$ & 0.2 & $-0.7^{* *}$ & $-0.6^{* *}$ & 0.3 & 0.1 & $-0.8^{* *}$ & $0.5^{*}$ & 0.1 \\
\hline & & & & & & & & & - & $0.8^{* *}$ & $0.9^{* * *}$ & $0.8^{* * *}$ & $0.7^{* * *}$ & -0.4 & $-0.7^{* *}$ & 0.4 & 0.2 & 0.1 & -0.2 & $0.5^{*}$ & 0.1 \\
\hline & & & & & & & & - & $-0.8^{* *}$ & $-0.8^{* *}$ & $-0.7^{* *}$ & $-0.9^{* *}$ & $-0.7^{* *}$ & $-0.6^{*}$ & 0.2 & $-0.8^{* *}$ & $-0.5^{*}$ & $-0.6^{* *}$ & 0.3 & 0.2 & $-0.6^{* *}$ \\
\hline & & & & & & & - & 0.3 & -0.2 & -0.3 & $-0.7^{* *}$ & -0.3 & 0.2 & -0.2 & -0.2 & 0.3 & -0.1 & -0.2 & $0.5^{*}$ & 0.1 & 0.1 \\
\hline & & & & & & - & $0.76^{* *}$ & $0.78^{* *}$ & $-0.8^{* *}$ & $-0.9^{* * *}$ & $-0.7^{* *}$ & $-0.8^{* *}$ & $-0.7^{* *}$ & $-0.7^{* * *}$ & $-0.7^{* *}$ & $-0.7^{* * *}$ & 0.3 & $0.7^{* *}$ & -0.1 & $-0.7^{* *}$ & 0.1 \\
\hline & & & & & - & $0.9^{* *}$ & 0.3 & $0.8^{* \prime}$ & $-0.8^{* *}$ & $-0.8^{* *}$ & $-0.8^{* *}$ & $-0.9^{* *}$ & $-0.7^{* *}$ & $-0.5^{*}$ & $0.7^{* *}$ & $-0.7^{* * *}$ & $-0.4^{*}$ & $-0.6^{* *}$ & $0.4^{*}$ & 0.3 & $-0.7^{*}$ \\
\hline & & & & - & $0.6^{*}$ & 0.2 & 0.4 & $0.6^{* *}$ & 0.3 & -0.4 & -0.5 & 0.5 & $-0.7^{* *}$ & $-0.6^{*}$ & 0.3 & -0.3 & -0.1 & $0.6^{* *}$ & 0.3 & $-0.7^{* *}$ & -0.2 \\
\hline & & & - & $0.9^{* *}$ & $0.9^{* * *}$ & 0.2 & 0.4 & $0.6^{* *}$ & 0.5 & $0.7^{* *}$ & $0.7^{* * *}$ & $0.7^{* *}$ & $0.6^{* *}$ & $0.9^{* *}$ & $0.6^{* *}$ & $0.5^{*}$ & $0.6^{* *}$ & $0.5^{*}$ & 0.2 & 0.2 & $0.6^{* *}$ \\
\hline & & - & 0.3 & 0.2 & 0.2 & 0.1 & 0.2 & 0.2 & 0.2 & 0.2 & 0.2 & 0.1 & 0.2 & 0.2 & 0.2 & 0.1 & 0.2 & 0.2 & $0.4^{*}$ & 0.3 & $0.7^{*}$ \\
\hline & - & 0.3 & $0.5^{*}$ & 0.2 & 0.3 & 0.2 & 0.3 & 0.3 & 0.3 & $0.65^{* *}$ & 0.2 & 0.3 & $0.6^{* * *}$ & 0.3 & 0.2 & $0.6^{* * *}$ & 0.3 & $0.5^{*}$ & 0.2 & 0.2 & 0.2 \\
\hline - & 0.5 & 0.2 & 0.3 & 0.3 & $0.7^{* *}$ & 0.3 & $0.8^{* *}$ & 0.5 & $0.7^{* *}$ & $0.8^{* *}$ & 0.3 & $0.82^{* *}$ & $0.7^{* *}$ & $0.7^{* *}$ & $0.7^{* *}$ & $0.6^{* *}$ & $0.9^{* *}$ & $0.7^{* *}$ & $0.9^{* * *}$ & $0.6^{* *}$ & $0.4^{*}$ \\
\hline
\end{tabular}

ns: Not Significant, *and** : Significant at $P=0.05$ and $P=0.01$ levels of probability, respectively. 
Water and mineral nutrient contents have been reported to increase with altitude and precipitation, thereby increasing essential oil content [24]. Effects of altitude on soil texture have been reported to affect a variety of physiological properties including the chlorogenic acid content of artichokes [9], thymol and carvacrol contents of thyme [25], artemisin content of Artemisia annua [10], antioxidant levels of vegetables [11], essential oil composition and antimicrobial properties of wild mint [12], and essential oil content of Origanum vulgare [26]. Of the morphological properties assessed by the present study, total dry matter, and leaf area indices, exhibited the greatest correlation with essential oil content, regardless of region and plant tissue (Table 6). Although many studies have compared the volatile oil contents of plants from various sites, none have investigated the effects of altitude on volatile oil content $[1,27]$. Here, we conclude that many essential oil components, (e.g., nonadecane), decrease with altitude, likely owing to two related factors. First many environmental factors (e.g., precipitation) are affected by altitude, wind, daily temperatures, and cloudiness. However, the amount of evaporation and mean temperature decreased with increasing altitude and had a better effect on the essential oil content and composition. Furthermore, the roots of $C$. arvense yielded the greatest essential oil content, regardless of region. It seems that soil depth increases with altitude and that plant metabolism, growth, development, and phytochemical levels are affected by the absorption and transition of essential nutrients [28]. However, morphological and physiological characteristics are also affected by altitude [29] and seasonal variation [30]. The depth of soils in Chelgerd region increased with increasing altitude and the content of many of components of essential oil was increased [9,13,27,31-33].

The results of the present study indicate that the essential oil composition of $C$. arvense is significantly affected by differences in climate and soil properties. The main components of $C$. arvense essential oil were nonadecane, $\beta$-citronellol, camphor, heneicosane and phytol, and the most levels of nonadecane and camphor were obtained from the roots of plants found in the Chelgerd region, whereas the most levels of $\beta$-citronellol were obtained from the leaves of plants found in the Chelgerd region, and the most levels of nonadecane and overall essential oil were obtained from the roots of plants found in the Chelgerd region. In addition, the present study also demonstrates that the essential oil components of $C$. arvense are affected by climate, soil and plant tissue. This study provides useful information regarding the efficacy of regions and plant tissues on the content and composition of essential oil in C. arvense.

\section{Acknowledgements}

This work was supported by the Islamic Azad University Branch of Shahrekord, Iran. We are very grateful to Mrs. Parvin Karimi from Education Center of Shahrekord for helpful advice for secondary reviewer.

\section{ORCID}

Narges Amiri: 0000-0002-7210-8235

Mehrab Yadegari: 0000-0002-3122-4686

Behzad Hamedi: 0000-0002-9022-9866

\section{References}

[1] A. Ghasemi Pirbalouti and M. Mohammadi (2013). Phytochemical composition of the essential oil of different populations of Stachys lavandulifolia Vahl, Asian Pac. J. Trop. Biomed. 3, 123-128.

[2] R.J. Moore (1975). The biology of Canadian weeds. 13: Cirsium arvense (L.) Scop, Canadian J. Plant Sci. 55, 1033-1048.

[3] J.K. Sciegienka, E.N. Keren and F.D. Menalled (2011). Impact of root fragment dimension, weight, burial depth, and water regime on Cirsium arvense emergence and Growth, Canadian J. Plant Sci. 91, 1027-1036.

[4] V.A. Bhattaram, U. Graefe, C. Kohlert, M. Veit and H. Derendorf (2002). Pharmacokinetics and bioavailability of herbal medicinal products, Phytomed. 9, 1-33. 
[5] K.N. Prashanth, S. Neelam and S. Chauhan (2006). Search for antibacterial and antifungal agents from selected Indian medicinal plants, J. Ethnopharm. 107, 182-188.

[6] L.B. Nadeau and W.H. Vanden Born (1989). The root system of Canada thistle, Canadian J. Plant Sci. 69, 1199-1206.

[7] B. Yildiz, T. Arabaci and T. Dirmenci (2013). Two new species of Cirsium (Asteraceae) and notes on allies from Turkey, Turk. J. Bot. 37, 1045-1054.

[8] H. Hajimehdipoor, A.R. Gohari and S. Saeidnia (2014). Comparative study of the total phenol content and antioxidant activity of some medicinal herbal extracts, Res. J. Pharm. 1, 21-25.

[9] M. Yadegari and S. Yousefi (2016). Effects of environmental conditions on morphological and physiological characters of Cynara scolymus, Bang. J. Bot. 45, 605-610.

[10] A. Omer (2008). Effect of soil type and seasonal variation on growth, yield, essential oil and artemisinin content of Artemisia annua L, Inter. Res. J. Hort. 1, 15-27.

[11] W. Kalt (2005). Effects of production and processing factors and major fruit and vegetables antioxidants, J. Food Sci, 70, 11-19.

[12] A.M. Viljoen, S. Petkar and S.F.A. Van Vuuren (2006). Chemo geographical variation in essential oil composition and the antimicrobial properties of Mentha longifolia in southern Africa, J. Essen. Oil Res. 18, 60-65.

[13] A. Gonuz and B. Özrgucu (1999). An investigation on the morphology, anatomy and ecology of Origanum onites L, Turk. J. Bot. 23, 19-32.

[14] S.P. Tiwari, P. Kumar, D. Yadav and D.K. Chauhan (2013). Comparative morphological, epidermal, and anatomical studies of Pinus roxburghii needles at different altitudes in the NorthWest Indian Himalayas, Turk. J. Bot. 37, 65-73.

[15] N. Zencirci (2008). Effect of upper plant parts on yield and quality in Turkish durum wheat landraces from different regions, altitudes, and provinces, Turk. J. Agri. Forest. 32, 29-39.

[16] T.D. Rendyuk, A.I. Shreter, V.L. Shelyuto and V.I. Glyzin (1977). A flavonoid glycoside from Cirsium arvense, I.M. Sechenov First Moscow Medical Institute, Translated from Khimiya Prirodnykh Soedinen ii, No 2, P. 282. Publishing corporation, 227 West $17^{\text {th }}$ street, New York, N.Y. 10011.

[17] V.L. Shelyuto, V.I. Glyzin and N.T. Bubon (1971). 3-O- methyl kaempferol from the flowers, Vitebsk Medical Institute, Translated from Khimiya Prirodnykh Soedinen ii, No 1, pp. 118-119. Publishing corporation, 227 West $17^{\text {th }}$ street, New York, N.Y. 10011.

[18] N. Theis (2006). Fragrance of Canada thistle (Cirsium arvense) attracts both floral herbivores and pollinators, J. Chem. Ecol. 32, 917-927.

[19] V. Mozaffarian (2008). A pictorial dictionary of botany botanical taxonomy Latin-EnglishFrench-Germany-Persian, Germany: Koeltz Scientific Books.

[20] R.P. Adams (2007). Quadruple Mass Spectra of Compounds Listed in Order of Their Retention Time on DB-5, In Identification of Essential Oil Components by Gas Chromatography/ Mass Spectroscopy. 4th ed, Carol Stream, IL 60188-2787. Allured publishing Corp, Carol Stream, USA.

[21] W.G. Taylor and C.E. Schreck (1985). Chiral-phase capillary gas chromatography and mosquito repellent activity of some oxazolidine derivatives of (+)- and (-)-citronellol, J. Pharm. Sci. 74,534-539.

[22] The Housekeeper's Almanac, or, the Young Wife's Oracle, for 1840 (1840). No. 134. NewYork: Elton, Print.

[23] C. Formisano, D. Rigano, F. Senatore, V.D. Feo, M. Bruno, and S. Rosselli (2007). Composition and allelopathic effect of essential oils of two thistles: Cirsium creticum (Lam.) D.'Urv. ssp. triumfetti (Lacaita) Werner and Carduus nutans L, J. Plant Interact. 2, 115-120.

[24] A. Maxwell and P. Jones (2007). Factors affecting the growth and secondary metabolism of medicinal plants, Mississippi State, North Mississippi Research and Extension Centre, $5421 \mathrm{~S}$ Hwy 145, Verona, MS 38879.

[25] A. Ghasemi Pirbalouti, M. Hashemi and F. Taherian Ghahfarokhi (2013). Essential oil and chemical compositions of wild and cultivated Thymus daenensis Celak and Thymus vulgaris L, Indus. Crop Prod. 48, 43-48.

[26] D. Vokou, S. Kokkini and J.M. Bessiere (1993). Geographic variation of Greek oregano (Origanum vulgare ssp) essential oils, Biochem. Sys. Ecol. 21, 287-295.

[27] A.C. Figuieredo, J.G. Barroso, L.G. Pedro and J.C. Scheffer (2008). Factors affecting secondary metabolite production in plants volatile compounds and essential oils, Flavour Frag. J. 23, 213226. 
[28] M. Yadegari (2017). Irrigation periods and Fe, $\mathrm{Zn}$ foliar application on agronomic characters of Borago officinalis, Calendula officinalis, Thymus vulgaris and Alyssum desertorum, Comm. Soil Sci. Plant Anal. 48, 307-315.

[29] D. Kremer, V. Matevski and V. Dunkic (2016). Essential oil contents and micro-morphological traits of Stachys iva Griseb. and S. horvaticii Micevski (Lamiaceae), Rec. Nat. Prod. 10, 228239.

[30] N. Gourine, M. Yousfi, I. Bombarda and B. Nadjemic (2010). Seasonal variation of chemical composition and antioxidant activity of essential oil from Pistacia atlantica Desf, leaves from Algeria, Indus. Crop Prod. 31, 203-208.

[31] K. Baczek, O. Kosavowska, L. Jaroslaw and Z. Przibil (2016). Accumulation of phenolic compounds in the purple betony herb (Stachys officinalis L.) originated from cultivation, Herbal. Bot. 62, 7-16.

[32] M. Yadegari and F. Mohmmadian (2015). Ecological diversity and phytochemical properties medicinal plant (Stachys pilifera) in Kohgiluyeh and BoyerAhmad province, Inter. J. Biol. Pharm. Allied Sci. 4, 182-188.

[33] B. Teixeira, A. Mrques, C. Ramos and C. Serrano (2013). Chemical composition and bioactivity of different oregano (Origanum vulgare) extract and essential oil, J. Sci. Food Agri. 93, 27072714.

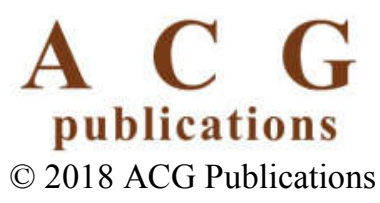

